\title{
Predictors of Chronic Postsurgical Pain in Elderly Patients Undergoing Hip Arthroplasty: A Multi-Center Retrospective Cohort Study
}

\author{
Yi Lu ${ }^{l}, *$ \\ Bang $\mathrm{Hu}^{2, *}$ \\ Haitao Dai ${ }^{3, *}$ \\ Bao Wang' \\ Jiayin $\mathrm{Yao}^{4}$ \\ Xinhua Yao'
}

'Department of Anesthesiology, The Affiliated TCM Hospital of Guangzhou Medical University, Guangzhou, Guangdong Province, People's Republic of China; ${ }^{2}$ Department of Colorectal Surgery, Guangdong Provincial Key Laboratory of Colorectal and Pelvic Floor Disease, The Sixth Affiliated Hospital of Sun Yat-Sen University, Guangzhou, Guangdong Province, People's Republic of China;

${ }^{3}$ Department of Interventional Radiology, The First Affiliated Hospital of Sun Yat-Sen University, Guangzhou, Guangdong Province, People's Republic of China; ${ }^{4}$ Department of Gastroenterology, Guangdong Provincial Key Laboratory of Colorectal and Pelvic Floor Disease, The Sixth Affiliated Hospital of Sun Yat-Sen University, Guangzhou, Guangdong Province, People's Republic of China

*These authors contributed equally to this work

Correspondence: Xinhua Yao Department of Anesthesiology, The Affiliated TCM Hospital of Guangzhou Medical University, 16th Zhuji Road, Guangzhou, Guangdong Province, 510130,

People's Republic of China

Tel +86 I3719289709

Email yxh200210@126.com

Jiayin Yao

Department of Gastroenterology, Guangdong Provincial Key Laboratory of Colorectal and Pelvic Floor Disease, The Sixth Affiliated Hospital of Sun Yat-Sen

University, 26th Yuancun Second Road,

Guangzhou, Guangdong Province, 510655,

People's Republic of China

Tel +86 I3826462890

Email yjyin@mail3.sysu.edu.cn
Background: Identifying patients at high risk of developing chronic postsurgical pain (CPSP) is of extreme importance in order to help launch appropriate therapeutic strategies and intensive initiation of pain management.

Aim: In this study, we aimed to conduct a multi-center retrospective cohort study to establish a prognostic model and a nomogram to predict the risks of CPSP in elderly patients who underwent hip arthroplasty at 6 months postoperatively.

Methods: We collected data from 736 patients aged $>65$ years who had undergone hip arthroplasty from October 1, 2016 to September, 30, 2018 at multiple tertiary referral centers in Guangzhou, China. All data were randomly stratified into a training set and a testing set at a ratio of 8:2. Data were analyzed via multiple logistic regression analysis with receiver operating characteristic (ROC) curves and areas under the curve. This model was further validated by estimating calibration and discrimination. A nomogram was ultimately developed.

Results: A total of 736 eligible patients were enrolled, $27.20 \%$ of whom developed CPSP within 6 months postoperatively. Preoperative pain in the surgical area $(\mathrm{OR}=2.456,95 \%$ CI:1.814-3.327, $\mathrm{P}<0.001)$, preoperative depression state $(\mathrm{OR}=1.256,95 \%$ CI:1.146-1.378, $\mathrm{P}<0.001)$, surgical type ( $\mathrm{OR}=7.138,95 \% \mathrm{CI}: 3.548-14.364, \mathrm{P}<0.001)$, acute postoperative numerical rating scale score $(\mathrm{OR}=5.537,95 \% \mathrm{CI}: 3.607-8.499, \mathrm{P}<0.001)$ and analgesic type (patient-controlled epidural analgesia: $\mathrm{OR}=0.129,95 \%$ CI:0.055-0.299, $\mathrm{P}<0.001$; patientcontrolled intravenous analgesia: $\mathrm{OR}=0.033,95 \% \mathrm{CI}: 0.011-0.097, \mathrm{P}<0.001)$ were identified as independent significant factors associated with CPSP. A prognostic model was established and further validated. An ROC curve confirmed the predictive ability of this model with a high sensitivity value of $92.12 \%$ (95\% CI:86.90-95.74) and specificity value of $91.72 \%$ (95\% CI:88.77-94.11). A nomogram was developed to simplify the use of the predictive model in clinical practice.

Conclusion: This prognostic model could be of great value in clinical practice, serving as the basis for early personalized analgesic management of elderly patients undergoing hip arthroplasty.

Keywords: chronic postsurgical pain, CPSP, prognostic model, nomogram, retrospective study

\section{Introduction}

There is an increasing number of elderly patients undergoing hip arthroplasty for the treatment of osteoarthritis and/or osteonecrosis of the femoral head (ONFH) with unbearable persistent pain. ${ }^{1}$ It was reported that the annual incident rate of 
total hip arthroplasty increased from 15.2/100,000 in 2010 to $22.3 / 100,000$ in 2018 in Korea, showing the same increased tendency as reports from Western countries. ${ }^{2}$ Previous studies have reported that approximately $27 \%$ of patients developed chronic postsurgical pain (CPSP) after hip arthroplasty. ${ }^{3}$ It is estimated that there is a higher incidence of CPSP in elder patients due to their poor functional recovery. ${ }^{4}$ CPSP can result in delayed rehabilitation, worsened functional disability, exacerbated pain-related depression or anxiety, lowered quality of life, and increased economic burden. Therefore, the identification of patients at high risk of CPSP, and correspondingly launching extensive therapeutic and pain strategies is of great importance.

Currently, the aetiology and pathophysiologic mechanisms behind CPSP are multifactorial. CPSP was reported to be associated with demographic factors, genetic polymorphisms, psychosocial status, surgery type, and pain itself. The presence of preoperative pain and acute postoperative pain (APOP) predicts the likelihood of developing CPSP. Since surgery is the main strategy used to relieve preoperative pain, anesthesiologists focus on managing APOP with various postoperative analgesia methods including patient-controlled epidural analgesia (PCEA), patient-controlled intravenous analgesia (PCIA), and oral analgesia (OA). However, predicting and managing CPSP are still challenges for clinical anesthesiologists. Undoubtedly, an effective and validated model that can predict the occurrence of CPSP is of desperate need. In the present study, we aimed to identify significant associated risk factors for CPSP, establish a validated prognostic model, and further develop an easy-to-use clinically applicable nomogram. To our knowledge, this is the first prognostic model for CPSP risk in elderly patients undergoing hip arthroplasty in China.

\section{Methods}

\section{Study Design}

In this multi-center retrospective cohort study, we collected data from 801 patients who had undergone hip arthroplasty between October 1, 2016 and September 30, 2018 at the Affiliated TCM Hospital of Guangzhou Medical University, Guangzhou Orthopedic Hospital, and Guangzhou Hospital of Integrated Traditional and Western Medicine. The inclusion criteria were as follows: patients aged $>65$ years; patients with a clear indication of hip arthroplasty, including osteoarthritis and ONFH; and patients administered a combined spinal and epidural anesthesia. The exclusion criteria were as follows: patients who suffered from severe cardiovascular, chronic respiratory, or central nervous system (CNS) diseases; patients addicted to drugs or were allergic to analgesic drugs; and patients administered antidepressants or sedative drugs. This manuscript adheres to the applicable STROBE guidelines. Ethical approval for this study (No.2019NK028) was provided by the Ethical Committee of the Affiliated TCM Hospital of Guangzhou Medical University, China (Chairperson Prof. Wen Xu). The study design has been registered online (ChiCTR1900025103). Requirement for written informed consent was waived by the Institutional Review Board due to a retrospective design and use of anonymous data. We securely protected the information on all the individuals and it was only made available to the investigators. This study was conducted in accordance with the Declaration of Helsinki.

\section{Outcome Measures and Definitions}

The main outcome was the occurrence of CPSP at 6 months postoperatively. Diagnosis of CPSP was made based on the guidelines of the International Society for Pain (IASP: ICD-11) as follows: ${ }^{5}$ postoperative occurrence of pain; pain continuing for more than three months; exclusion of chronic infection or malignancy as pain sources; different type of pain compared with the original pre-surgery pain. Patients were telephone interviewed by an anesthesiologist (YL) to assess numerical rating scale (NRS) scores according to their pain density in the surgical area at 6 months postoperatively. Numerical rating scales (NRS) range from 0 to 10 , with 0 representing no pain and 10 representing the worst imaginable pain. The cut-off score for CPSP was set at NRS $\geq 3,{ }^{6}$ referring to mild and severe pain.

\section{Data Acquisition}

Data from eligible patients were collected at the following time points: preoperative, intraoperative, 24-72 $\mathrm{h}$ postoperatively, and 6 months postoperatively. Preoperative variables included sex, age, body mass index (BMI), smoking, drinking, education, American Society of Anesthesiologists (ASA) grading, ${ }^{7}$ preoperative depression and anxiety scores, presence of any preoperative chronic pain in the non-surgical area, and preoperative pain in the surgical area. ASA grading was conducted as previously described. ${ }^{8}$ Preoperative depression scores were evaluated using the Center for Epidemiological 
Studies-Depression Scale (CES-D), ${ }^{9}$ which consisted of 20 items involving symptoms such as depressed mood, helplessness, worthlessness, psychomotor retardation, and eating and sleeping problems $(\mathrm{CES}-\mathrm{D}=0-60$, higher scores indicated greater depression). Preoperative anxiety scores were assessed using the Pain Anxiety Symptoms ScaleShort Form (PASS-20), ${ }^{10}$ which consisted of 20 items measuring anxiety-related feelings, behavior, and physical sensations (PASS-20 $=0-100$, higher scores indicated greater anxiety). Both CES-D and PASS-20 have ideal internal consistencies $(\alpha=0.85-0.90$ and $\alpha=0.81$, respectively) and strong construct validities. ${ }^{9}$ The presence of any preoperative chronic pain was defined as a dichotomous yes or no. Preoperative pain in the surgical area was defined as NRS scores in the areas including side of hip, lower limb and waist. Data such as ASA grading, CES-D scores and PASS-20 scores were evaluated and recorded by anesthesiologists before the surgeries. All the preoperative data were collected from hosptial's electronic database.

Intraoperative variables included surgery type, surgery duration, blood loss and transfusion volume, and use of dexmedetomidine during surgery. The two main surgery types were total-hip and semi-hip replacements. All patient data were obtained through medical records, and verified by the second anesthesiologist when uncertain data are encountered during data collection.

Acute postoperative variables included analgesic methods and APOP. Analgesic methods were divided into three types according to analgesia type. Patients administrated PCIA received an intravenous opioid analgesic of $100 \mathrm{~mL}$ standard opioid concentration containing $0.8 \mathrm{mg}$ fentanyl and $4 \mathrm{mg}$ droperidol, while those with PCEA application received epidural infusion of $100 \mathrm{~mL}$ standard opioid concentrations containing $0.2 \mathrm{mg}$ fentanyl, $150 \mathrm{mg}$ ropivacaine and $4 \mathrm{mg}$ droperidol using a disposable analgesia pump (100 mL, WZ-6523C-4) with a continuous basal rate of $2 \mathrm{~mL} / \mathrm{h}$ and bolus dose of $0.5 \mathrm{~mL}$ at each 15 -minute time interval. Patients given an OA received only diclofenac sodium at a dose of $75 \mathrm{mg}$ at intervals of 24 h. Anesthesiologists interviewed patients $48-72 \mathrm{~h}$ after surgery to assess APOP when moving using the NRS. If a patient was not able to express themselves at 24 hours promptly after surgery, we assessed APOP at a narrow time gap from $48 \mathrm{~h}$ to $72 \mathrm{~h}$ postoperatively.

All patients underwent phone interviews with an anesthesiologist to collect NRS scores. Patients with a NRS $\geq 3$ at 6 months postoperatively were defined as CPSP.

\section{Statistical Analysis}

Continuous variables are presented as mean \pm standard deviation (SD), while categorical variables are presented as percentages or proportions. Continuous variables were analyzed using independent-sample t-tests as appropriate, while categorical variables were analyzed using the Chisquare or Fisher's exact test. We randomly stratified all the data into a training set and a testing set at a ratio of 8:2. All cases were allocated a random number generated by SPSS (version 22.0, IBM Corp., Armonk, NY). The range of random numbers was set from 0 to 100 ; cases with numbers ranging from 0 to 80 were assigned to the training set, and those with numbers ranging from 80.0001 to 100 were assigned to the testing set. Our random seed was 666. The statistical analysis could easily be repeated using the same random seed. Observation independence, absence of multicollinearity, and sufficient sample size were confirmed to meet the assumptions for conducting logistic regression. Multiple logistic regression analysis was conducted to establish a forward stepwise model with receiver operating characteristic (ROC) curves constructed and areas under the curve (AUC) calculated. The results were then confirmed using a backward elimination procedure. The model was eventually validated with calibration using the Hosmer-Lemeshow goodness-of-fit test, and discrimination was assessed using AUC. A nomogram was established using $\mathrm{R}$ software ( $\mathrm{R}$ Foundation for Statistical Computing, Vienna, Austria). A two-tailed P-value of $<0.05$ was considered to be statistically significant. All statistical analyses were performed using IBM SPSS. The statistical methods used in this study were reviewed by Jinxin Zhang from the Department of Medical Statistics, University of Sun Yat-Sen.

\section{Results \\ Patient Characteristics}

We excluded $13(1.62 \%)$ patients due to severe postoperative complications, including pulmonary embolism $(n=8)$ and myocardial infarction $(n=5)$. Furthermore, we excluded $4(0.50 \%)$ patients due to severe allergy. Finally, we excluded $18(2.25 \%)$ patients due to loss of follow-up and $30(3.75 \%)$ patients due to incomplete follow-up data. Figure 1 presents a flow chart according to the CONSORT statement showing the progress of the 
participants through the study. Ultimately, we included 736 patients (follow-up rate $=91.89 \%$ ) in the final analysis. Table 1 presents details of the included patients' baseline characteristics.

\section{Factors Associated with CPSP at 6 Months Postoperatively}

A total of 200 patients $(27.20 \%)$ were diagnosed with CPSP at 6 months postoperatively. CPSP was significantly associated with preoperative pain in the surgical area ( $\mathrm{OR}=2.456,95 \% \mathrm{CI}: 1.814-3.327, \mathrm{P}<0.001)$, preoperative depression state (CES-D) (OR=1.256, 95\% CI:1.146$1.378, \quad \mathrm{P}<0.001)$, surgical type $(\mathrm{OR}=7.138, \quad 95 \%$ CI:3.548-14.364, $\mathrm{P}<0.001$ ), acute postoperative NRS score $(\mathrm{OR}=5.537,95 \% \mathrm{CI}: 3.607-8.499, \mathrm{P}<0.001)$, and analgesic type (PCEA: OR=0.129, 95\% CI:0.055-0.299, $\mathrm{P}<0.001 ; \quad$ PCIA: $\quad \mathrm{OR}=0.033, \quad 95 \% \quad$ CI:0.011-0.097, $\mathrm{P}<0.001$; Table 2). Absolute risk changes for type of surgery (hemi-hip vs total-hip), analgesic method (OA vs PCEA), and analgesic method (OA vs PCIA) is 0.249 (95\% CI: $0.181-0.315), 0.538$ (95\% CI: $0.456-0.609)$, and 0.596 (95\% CI: $0.520-0.660)$, respectively.

\section{Prognostic Model Appraisement}

The prognostic model was established as follows:

$$
P_{\mathrm{CPSP}}=\frac{e^{\text {index }}}{1+e^{\text {index }}}
$$

where prognostic

index $=-13.049+1.965 \times x_{2}+0.899 \times x_{2}+0.228 \times x_{4}-$ $2.051 \times \mathrm{I}\left[x_{5}=\mathrm{PCEA}\right]-3.418 \times \mathrm{I}\left[x_{5}=\right.$ PCIA $]$

( $\mathrm{X}_{1}=$ type of surgery (total or hemi-hip replacement); $\mathrm{X}_{2}$ =preoperative NRS in the surgical area; $X_{3}=$ acute postoperative NRS; $\mathrm{X}_{4}=$ preoperative depression state [CES$\mathrm{D}] ; \mathrm{X}_{5}=$ analgesic type [PCEA or PCIA]).

ROC curve was generated to evaluate the predictive ability of this model. The AUC was 0.968 (95\% CI: $0.955-0.980, \quad \mathrm{P}<0.001)$ with a sensitivity value of $92.12 \%$, specificity value of $91.72 \%$, positive predictive value of $80.42 \%$, negative predictive value of $96.93 \%$, positive likelihood ratio of 11.13 , and negative likelihood ratio of 0.09 (Figure 2A). The prognostic model was subsequently applied to the testing cohort. Discrimination of the model was acceptable, which further indicated a good predictive power and discriminatory ability (Figure 2B). Regarding calibration, the Hosmer-Lemeshow goodness- of-fit test indicated that this model showed a good fit with a high level of agreement between the calculated risk and the observed outcomes (Figure 3).

\section{Prognostic Nomogram for CPSP}

All the significant associated factors, including the protective and risk factors, were integrated in the establishment of nomogram (Figure 4). The probability of CPSP was easily obtained by summing total points and matching vertically downward to the risk of CPSP.

\section{Discussion}

In this study, preoperative pain in the surgical area, preoperative depression state, surgical type, acute postoperative pain, and analgesic type were confirmed to be the independent risk factors for CPSP in elderly patients at 6 months after hip arthroplasty. A prognostic model predicting CPSP is of great use in therapeutic strategies launching.

Standardized definition of CPSP was presented in the International Classification of Disease Eleventh Reversion (ICD-11) as pain occurring for more than 3 months postoperatively with other causes of pain excluded. ${ }^{5,11}$ According to Sugiyama et al, the prevalence of CPSP is approximately 5-65\%, which varies for different surgery types. ${ }^{12}$ A recent cross-sectional survey of 2043 patients who had undergone surgery showed that among them, $40.4 \%$ reported chronic pain, $18.3 \%$ reported moderate-tosevere pain, and $24.5 \%$ reported sensory abnormalities. ${ }^{13}$ Regarding hip arthroplasty, a high CPSP rate of $27 \%$ has been reported; however, the data still remain unclear for elderly patients. ${ }^{14}$ CPSP is a chronic pain condition with limited therapeutic strategies compared with those for APOP. ${ }^{15-17}$ Patients with CPSP endure significant suffering, which might eventually result in depression, anxiety, and other psychological problems. Consequently, this generates a huge economic burden and large consumption of medical resources. ${ }^{18,19}$ It is increasingly recognized that good management of CPSP will definitely improve postoperative recovery and clinical outcomes, ${ }^{11,20,21}$ which should draw the attention of anaesthesiologists.

A number of putative risk factors may contribute to CPSP development. ${ }^{13}$ Several predictive factors of CPSP have been reported, including genetic background ${ }^{22,23}$ and pain history ${ }^{24,25}$ and psychological factors. ${ }^{26,27}$ In this retrospective study, we identified significant risks factors 


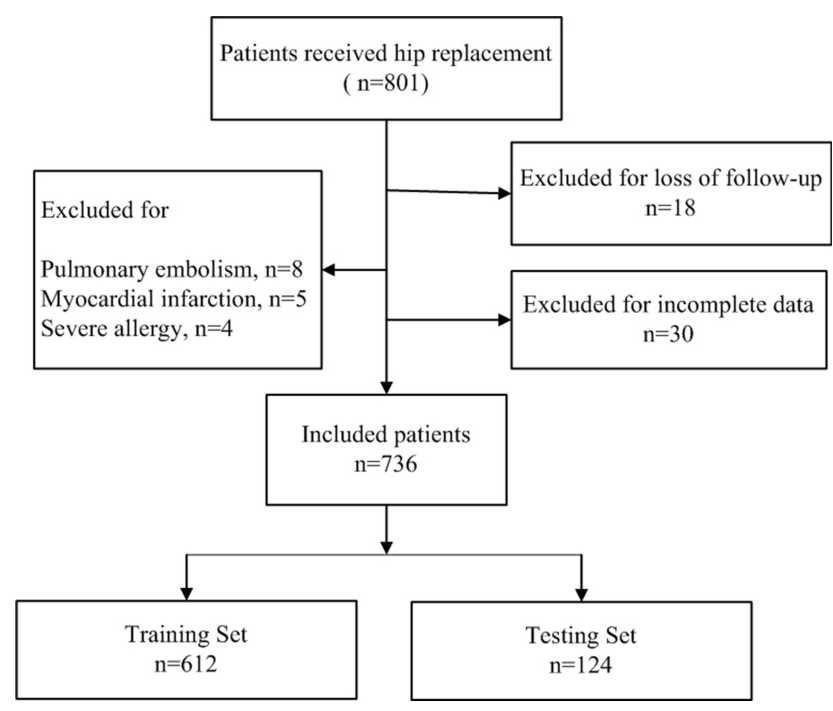

Figure I Study cohort flowchart.

associated with CPSP to be preoperative NRS in the surgical area, preoperative depression status, surgery type, postoperative analgesic method, and acute postoperative NRS. We combined these factors in a prognostic model and developed a nomogram to facilitate CPSP risk prediction in clinical practice. This model was based solely on clinical and demographic variables, making it easily and broadly applicable to clinical practice. For patients with a high risk of CPSP, it is crucial to launch preemptive analgesia preoperatively that is continued postoperatively.

Both PCEA and PCIA are widely used analgesia methods in APOP management, with confirmed analgesic effects on elderly patients undergoing hip replacement. Regarding CPSP, reports on the chronic analgesic effects of PCEA and PCIA remain controversial. ${ }^{28-30}$ Our findings indicate that both PCEA and PCIA have a protective effect against CPSP. Lowering the pain threshold and minimizing APOP might be the probable mechanism.

Moreover, preoperative NRS score in the surgical area and acute postoperative NRS score were found to be significant risk factors and predictive factors for CPSP. ${ }^{31}$ The underlying pathogenesis of CPSP could be attributed to the imbalance of inflammatory cytokines due to surgery stress, which sensitizes the central nervous system (CNS). Preoperative pain and acute postoperative pain are strongly related to CPSP by sensitizing the peripheral nervous system and CNS. ${ }^{27,32}$ Effective management of preoperative and acute postoperative pain have been
Table I Patient Baseline Characteristics ( $N=736)$

\begin{tabular}{|c|c|}
\hline Characteristics & \%/Mean士SD \\
\hline Male/female (\%) & $44.29 / 55.71$ \\
\hline Age at diagnosis, years (mean $\pm \mathrm{SD})$ & $75.74 \pm 8.21$ \\
\hline Drink/not drink (\%) & $14.67 / 85.33$ \\
\hline Smoke/not smoke (\%) & $23.91 / 76.09$ \\
\hline Body mass index at diagnosis $\left(\mathrm{kg} / \mathrm{m}^{2}\right)(\operatorname{mean} \pm \mathrm{SD})$ & $22.15 \pm 2.43$ \\
\hline Use of DEX during surgery (No vs Yes) (\%) & $58.70 / 41.30$ \\
\hline Duration of surgery $(h)($ mean $\pm S D)$ & $2.69 \pm 0.93$ \\
\hline Blood loss volume $(\mathrm{mL})($ mean $\pm \mathrm{SD})$ & $358.08 \pm 188.84$ \\
\hline Blood transfusion volume $(\mathrm{U})($ mean $\pm \mathrm{SD})$ & $0.30 \pm 0.74$ \\
\hline \multicolumn{2}{|l|}{ Education (\%) } \\
\hline Below middle school & 48.64 \\
\hline High school & 48.23 \\
\hline Above college & 3.13 \\
\hline \multicolumn{2}{|l|}{ ASA grade (\%) } \\
\hline 1 & 36.96 \\
\hline II & 43.89 \\
\hline III & 19.15 \\
\hline \multicolumn{2}{|l|}{ Analgesic type (\%) } \\
\hline OA & 34.38 \\
\hline PCEA & 33.02 \\
\hline PCIA & 32.60 \\
\hline \multicolumn{2}{|l|}{ Type of surgery (\%) } \\
\hline Hemi-hip replacement & 47.69 \\
\hline Total hip replacement & 52.31 \\
\hline Preoperative NRS (surgical area) (mean \pm SD) & $2.43 \pm 1.14$ \\
\hline Preoperative NRS (non-surgical area) (mean \pm SD) & $0.16 \pm 0.37$ \\
\hline Acute NRS postoperatively (mean $\pm S D$ ) & $3.06 \pm 1.26$ \\
\hline Preoperative PASS-20 (mean \pm SD) & $31.16 \pm 12.04$ \\
\hline Preoperative CES-D (mean \pm SD) & $14.69 \pm 5.49$ \\
\hline CPSP at 6 months postoperatively (\%) & $27.17 / 72.83$ \\
\hline
\end{tabular}

Abbreviations: CPSP, chronic postsurgical pain; PCEA, patient-controlled epidural analgesia; PCIA, patient-controlled intravenous analgesia; OA, oral analgesia; NRS, numerical rating scale; ASA, American Society of Anesthesiologists; CES-D, Center for Epidemiological Studies-Depression Scale; PASS-20, Pain Anxiety Symptoms Scale-Short Form-20.

reported to prevent CNS sensitization and subsequently reduce the risks of CPSP. ${ }^{33-35}$

It is apparent that surgery itself also plays an important role. However, unlike other injuries, surgery allows the 
Table 2 Factors Associated with the Possibility of CPSP at 6 Months Postoperatively $(\mathrm{N}=6 \mathrm{I} 2$. Five Independent Variables Including Type of Surgery, Preoperative NRS in the Surgical Area, Acute Postoperative NRS, Preoperative Depression State, and Analgesic Type Were Involved in Final Regression Model)

\begin{tabular}{|c|c|c|c|c|c|c|}
\hline \multirow[t]{2}{*}{ Factors } & \multicolumn{3}{|c|}{ Univariate Analysis } & \multicolumn{3}{|c|}{ Multiple Logistic Regression } \\
\hline & OR & $95 \% \mathrm{Cl}$ & $P$ & OR & $95 \% \mathrm{Cl}$ & $P$ \\
\hline Gender (Male vs Female) & 1.055 & $0.737-1.512$ & 0.769 & & & \\
\hline Age & 0.979 & $0.958-1.001$ & 0.066 & & & \\
\hline BMI $\left(\mathrm{kg} / \mathrm{m}^{2}\right)$ & 1.021 & $0.950-1.098$ & 0.571 & & & \\
\hline \multicolumn{7}{|l|}{ Education(Ivs.2/3) } \\
\hline High school & 1.108 & $0.772-1.589$ & 0.579 & & & \\
\hline Above college & 0.291 & $0.066-1.277$ & 0.102 & & & \\
\hline \multicolumn{7}{|l|}{ ASA grade (Ivs.II/III) } \\
\hline II & 1.327 & $0.886-1.988$ & 0.170 & & & \\
\hline III & 1.049 & $0.629-1.749$ & 0.854 & & & \\
\hline Smoking (No vs Yes) & 1.034 & $0.685-1.559$ & 0.875 & & & \\
\hline Alcohol abuse (No vs Yes) & 1.406 & $0.87 I-2.270$ & 0.163 & & & \\
\hline Use of DEX during surgery (No vs Yes) & 0.831 & $0.578-1.194$ & 0.317 & & & \\
\hline Blood loss volume $(\mathrm{mL})$ & 1.001 & $1.000-1.002$ & 0.094 & & & \\
\hline Blood transfusion volume $(\mathrm{mL})$ & 1.174 & $0.937-1.47 \mid$ & 0.163 & & & \\
\hline Duration of surgery $(h)$ & 1.090 & $0.904-1.316$ & 0.367 & & & \\
\hline Preoperative NRS (surgical area) & 1.706 & $1.448-2.009$ & $<0.001$ & 2.456 & $1.814-3.327$ & $<0.001$ \\
\hline Preoperative NRS (non-surgical area) & 1.025 & $0.627-1.675$ & 0.922 & & & \\
\hline Preoperative PASS-20 & 0.996 & $0.981-1.011$ & 0.602 & & & \\
\hline Preoperative CES-D & 1.115 & $1.065-1.168$ & $<0.001$ & 1.256 & $1.146-1.378$ & $<0.001$ \\
\hline Type of surgery (Hemi-hip vs Total hip) & 3.873 & $2.609-5.749$ & $<0.001$ & 7.138 & $3.548-14.364$ & $<0.001$ \\
\hline \multicolumn{7}{|l|}{ Analgesic method (OA vs PCEA/PCIA) } \\
\hline PCEA & 0.062 & $0.036-0.107$ & $<0.001$ & 0.129 & $0.055-0.299$ & $<0.001$ \\
\hline PCIA & 0.024 & $0.011-0.051$ & $<0.001$ & 0.033 & $0.011-0.097$ & $<0.001$ \\
\hline Acute NRS postoperatively & 6.517 & $4.789-8.869$ & $<0.001$ & 5.537 & $3.607-8.499$ & $<0.001$ \\
\hline
\end{tabular}

Abbreviations: CPSP, chronic postsurgical pain; PCEA, patient-controlled epidural analgesia; PCIA, patient-controlled intravenous analgesia; OA, oral analgesia; NRS, numerical rating scale; ASA, American Society of Anesthesiologists; DEX, dexmedetomidine.

identification and prediction of precise injuries and the ensuing pain. Therefore, it is necessary to identify these factors before and during surgery to predict the recovery course. Patients undergoing total hip arthroplasty might have a longer surgery duration, larger blood loss volume, and higher possibility of blood transfusion compared with those undergoing hemi-hip replacement. The more complicated the surgery is, the higher the CPSP prevalence.

Chronic persistent pain is regarded as a sociops ychobiomedical issue focusing on the importance of preoperative psychological status involving anxiety, depression, psychological vulnerability and flexibility. In this study, we identified preoperative depression as an independent risk factor for CPSP, which is consistent with previous studies. ${ }^{36,37}$ Patients with depression are supposedly more sensitized to postoperative discomfort and exaggerate the seriousness of painful sensations. Effective postoperative analgesia combined with appropriate psychological counseling could be of benefit. 

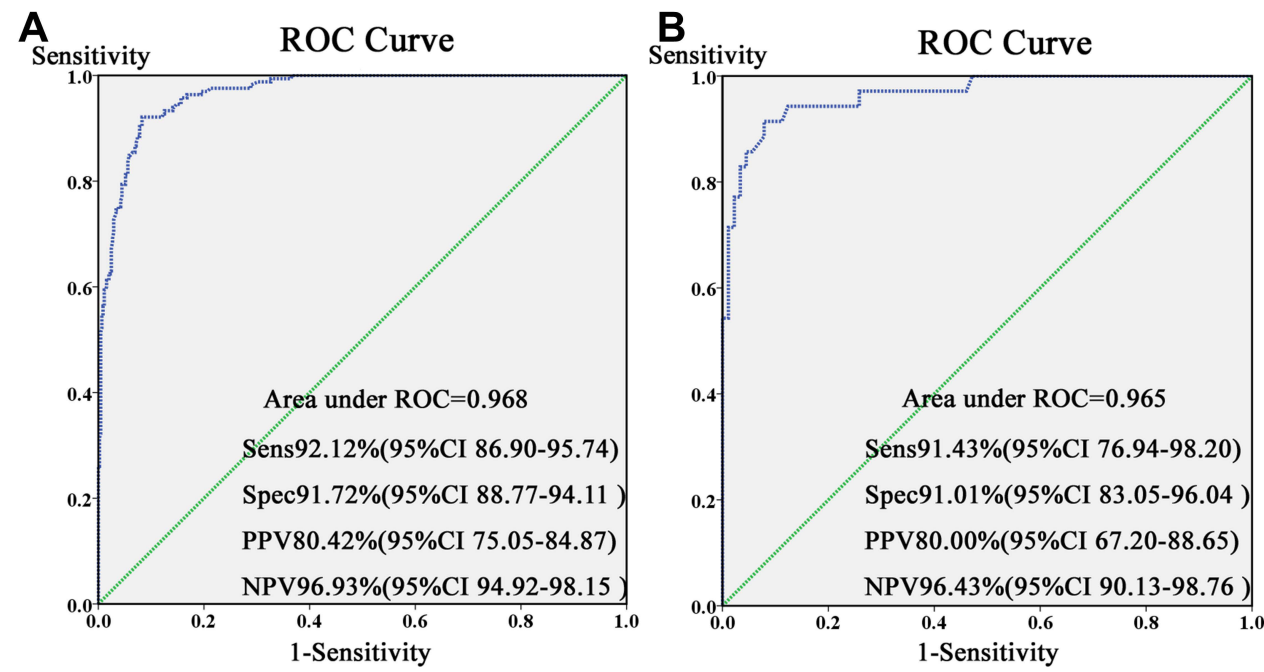

Figure 2 Receiver operating characteristic curve of the training and testing data. (A) The predictive ability of this model was appraised with an AUC of 0.968 , sensitivity of $92.12 \%$, and specificity of $91.72 \%$. (B) The estimated discrimination of the validated model was an AUC of 0.965 , sensitivity of $91.43 \%$, and specificity of $91.01 \%$.

Abbreviations: PPV, positive predictive value; NPV, negative predictive value.

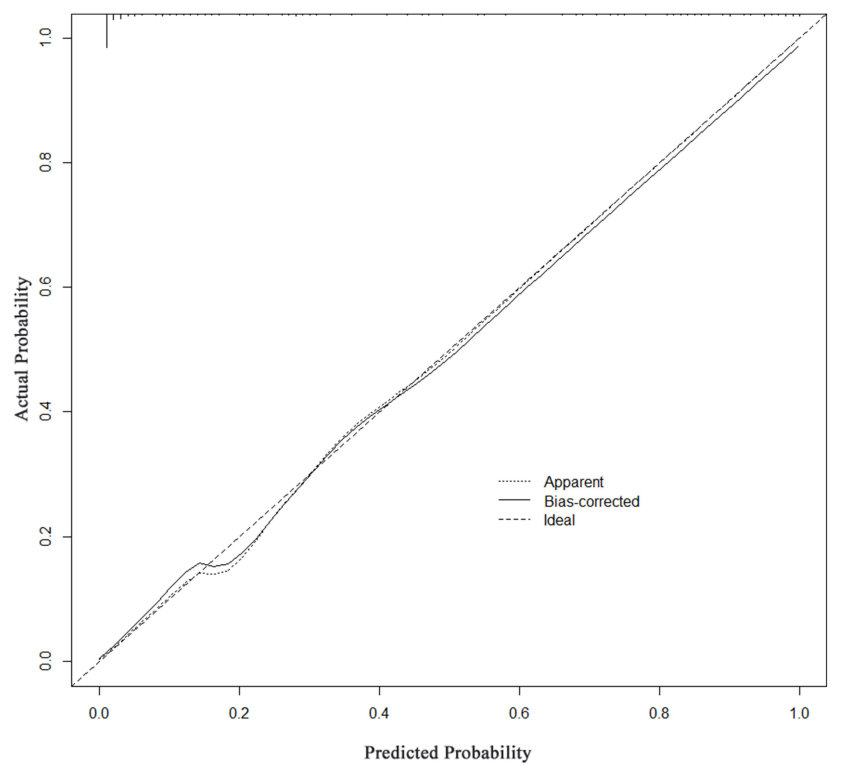

Figure 3 Hosmer-Lemeshow goodness-of-fit test $(P=I)$ demonstrated a good fit of this model.

Chronic pain is a major issue affecting more than $50 \%$ of the elderly population. ${ }^{38}$ Cognitive impairment makes pain assessment difficult, while medications acting on peripheral sensitization are less efficient. Moreover, experimental data on age-related changes in pain perception are scare and contradictory. ${ }^{39}$ Thus, pain management in the elderly is extremely challenging, especially for those who have undergone hip arthroplasty, functional recovery and pain relief is strongly demanded.
Currently, according to the procedure-specific pain management protocols in China, it is important to predict high CPSP risk in elderly patients and to initiate personalized treatment to minimize or prevent CPSP. Our study has several strengths. First, we enrolled a relatively large number of patients from multiple clinical centers with complete follow-up data. Given the size of our cohort and center, it is possible that the cohort presented here could approximate the national cohort. Further, we developed a nomogram, which is a simplified visual tool that allows for the convenient application of this model in clinical practice.

This study has several limitations that should be considered. First, we did not assess genetic factors. Further, the pain threshold varies across different races whereas this study only collected data from Chinese individuals. Lastly, we used data-driven rather than theory-driven analysis. Therefore, more generalizable findings can be obtained by analyzing data across different races.

In conclusion, we found that preoperative pain in the surgical area, preoperative depression state, surgical type, acute postoperative pain, and analgesic type were independent risk factors for CPSP in elderly patients undergoing hip arthroplasty. We established and validated a prognostic model with ideal sensitivity and specificity values and a nomogram to facilitate clinical application to serve as a basis for personalized pain management. 


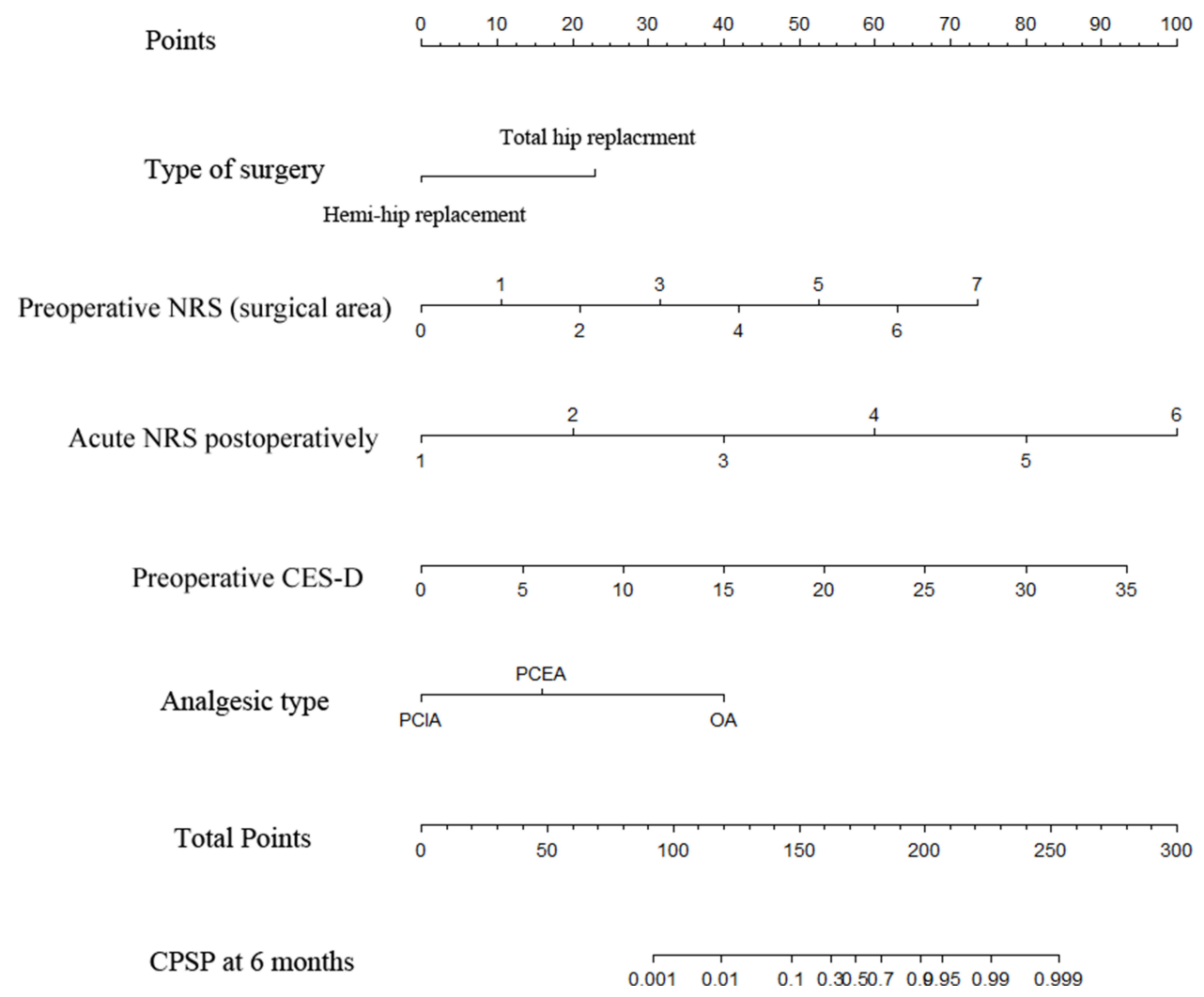

Figure 4 Prognostic nomogram for chronic postsurgical pain.

\section{Abbreviations}

APOP, acute postoperative pain; CPSP, chronic postsurgical pain; PCEA, patient-controlled epidural analgesia; PCIA, patient-controlled intravenous analgesia; OA, oral analgesia; NRS, numerical rating scale; IQR, interquartile range; $\mathrm{SD}$, standard deviation; $\mathrm{ROC}$, receiver operating characteristic; AUC, area under the curve; CNS, central nervous system; PPV, positive predictive value; NPV, negative predictive value; CES-D, Center for Epidemiological Studies-Depression Scale; PASS-20, Pain Anxiety Symptoms Scale-Short Form-20.

\section{Data Sharing Statement}

The datasets generated and analyzed during the current study are available from the corresponding authors or reasonable requests.

\section{Ethics Approval and Consent to Participate}

Ethical approval for this study (No.2019NK028) was provided by the Ethical Committee of the Affiliated TCM Hospital of Guangzhou Medical University, China (Chairperson Prof. Wen Xu). The study design has been registered online (ChiCTR1900025103, date of registration: August 11st 2019). Requirement for written informed consent was waived by the Institutional Review Board since it was a retrospective study using anonymous data.

\section{Acknowledgments}

The authors thank Professor Jinxin Zhang and Professor Zhuochen Lin, who are from the Department of medical statistics, University of Sun Yat-Sen University, No.74, Zhongshan Second Road, Yuexiu District, Guangzhou (510080), China for assistance in statistical analysis. Moreover, the authors thank Professor Jingwei Cui (Department of Anesthesiology, Family Planning Research Institute of Guangdong Province), Professor Jinhui Yang (Department of Anesthesiology, Guangzhou Orthopedic Hospital) and Professor Zhaohui Qing (Department of Anesthesiology, Guangzhou Hospital of Integrated Traditional and Western Medicine) for assistance in data acquisition.

\section{Author Contributions}

All authors contributed to data acquisition, analysis and interpretation, reviewed and agreed on the final version of 
article and the submitted journal, and were accountable for the contents of the article. YL, BH, and HTD-Conception and design, Data analysis and interpretation, Manuscript writing, Final approval of manuscript; JYY and XHYAdministrative support, Conception and design, Manuscript reviewing and revision, Final approval of manuscript; BW-Data acquisition and analysis, Manuscript revision, Final approval of manuscript.

\section{Funding}

The work was supported by grants from the National Natural Science Foundation of China (No.81900490), State Administration of Traditional Chinese Medicine of the People's Republic of China (Guangdong Province, No. 20201257), and Key Project of Affiliated TCM Hospital of Guangzhou Medical University (2020ZD04). The funders had no role in the study design, data collection and analysis, decision to publish or preparation of the manuscript.

\section{Disclosure}

The authors declare that they have no competing interests or disclosures.

\section{References}

1. Andronic O, Hincapie CA, Burkhard MD, et al. Lack of conclusive evidence of the benefit of biologic augmentation in core decompression for nontraumatic osteonecrosis of the femoral head: a systematic review. Arthroscopy. 2021. doi:10.1016/j.arthro.2021.04.062

2. Park JW, Won SH, Moon SY, Lee YK, Ha YC, Koo KH. Burden and future projection of revision total hip arthroplasty in South Korea. BMC Musculoskelet Disord. 2021;22(1):375. doi:10.1186/s12891021-04235-3

3. Kim DH, Pearson-Chauhan KM, McCarthy RJ, Buvanendran A. Predictive factors for developing chronic pain after total knee arthroplasty. J Arthroplasty. 2018;33(11):3372-3378. doi:10.1016/j. arth.2018.07.028

4. Schug SA, Bruce J. Risk stratification for the development of chronic postsurgical pain. Pain Rep. 2017;2(6):e627. doi:10.1097/ PR9.0000000000000627

5. Kehlet H, Jensen TS, Woolf CJ. Persistent postsurgical pain: risk factors and prevention. Lancet. 2006;367(9522):1618-1625. doi:10.1016/S0140-6736(06)68700-X

6. Lempa M, Koch G, Neugebauer E, Kohler L, Troidl H. [How much pain is tolerable? Target expectations of surgical patients for pain therapy]. Der Chirurg. 2000;71(10):1263-1269. German. doi: $10.1007 / \mathrm{s} 001040051213$

7. Hackett NJ, De Oliveira GS, Jain UK, Kim JY. ASA class is a reliable independent predictor of medical complications and mortality following surgery. Int $J$ Surg. 2015;18:184-190. doi:10.1016/j. ijsu.2015.04.079

8. Magi E. ASA classification and perioperative variables as predictors of postoperative outcome. Br J Anaesth. 1997;78(2):228. doi:10.1093/ $\mathrm{bja} / 78.2 .228$

9. Radloff LS. The CES-D scale: a self-report depression scale for research in the general population. Appl Psychol Meas. 1977;1 (3):385-401. doi:10.1177/014662167700100306
10. McCracken LM, Dhingra L. A short version of the Pain Anxiety Symptoms Scale (PASS-20): preliminary development and validity. Pain Res Manage. 2002;7(1):45-50. doi:10.1155/2002/517163

11. Huang CC, Sun WZ, Wong CS. Prevention of chronic postsurgical pain: the effect of preventive and multimodal analgesia. Asian J Anesthesiol. 2018;56(3):74-82.

12. Sugiyama Y, Iida H, Amaya F, et al. Prevalence of chronic postsurgical pain after thoracotomy and total knee arthroplasty: a retrospective multicenter study in Japan (Japanese Study Group of Subacute Postoperative Pain). J Anesth. 2018;32(3):434-438. doi:10.1007/s00540-018-2481-0

13. Johansen A, Romundstad L, Nielsen CS, Schirmer H, Stubhaug A. Persistent postsurgical pain in a general population: prevalence and predictors in the Tromso study. Pain. 2012;153(7):1390-1396. doi:10.1016/j.pain.2012.02.018

14. Pinedo-Villanueva R, Khalid S, Wylde V, Gooberman-Hill R, Soni A, Judge A. Identifying individuals with chronic pain after knee replacement: a population-cohort, cluster-analysis of Oxford knee scores in 128,145 patients from the English National Health Service. BMC Musculoskelet Disord. 2018;19(1):354. doi:10.1186/s12891-018-2270-9

15. Ko YK. Can chronic postsurgical pain be prevented by using regional block? Korean J Anesthesiol. 2019;72(4):295-296. doi:10.4097/ kja.19279

16. Jian W, Rejaei D, Shihab A, Alston TA, Wang J. The role of multimodal analgesia in preventing the development of chronic postsurgical pain and reducing postoperative opioid use. J Opioid Manag. 2018;14(6):453-461. doi:10.5055/jom.2018.0478

17. Steyaert A, Lavand'homme P. Prevention and treatment of chronic postsurgical pain: a narrative review. Drugs. 2018;78(3):339-354. doi:10.1007/s40265-018-0866-x

18. Aternali A, Slepian PM, Clarke H, et al. Presurgical distress about bodily sensations predicts chronic postsurgical pain intensity and disability 6 months after cardiothoracic surgery. Pain. 2021. doi:10.1097/j.pain.0000000000002325

19. Sahin F, Beyaz SG, Karakus N, Inanmaz ME. Total knee arthroplasty postsurgical chronic pain, neuropathic pain, and the prevalence of neuropathic symptoms: a prospective observational study in Turkey. $J$ Pain Res. 2021;14:1315-1321. doi:10.2147/JPR.S293856

20. Kissin I, Gelman S. Chronic postsurgical pain: still a neglected topic? $J$ Pain Res. 2012;5:473-489. doi:10.2147/JPR.S35145

21. Patzkowski MS, Patzkowski JC. Perioperative pain management and avoidance of long-term opioid use. Sports Med Arthrosc. 2019;27 (3):112-118. doi:10.1097/JSA.0000000000000244

22. Chidambaran V, Pilipenko V, Jegga AG, Geisler K, Martin LJ. Systems biology guided gene enrichment approaches improve prediction of chronic post-surgical pain after spine fusion. Front Genet. 2021;12:594250. doi:10.3389/fgene.2021.594250

23. Chidambaran V, Zhang X, Pilipenko V, et al. Methylation quantitative trait locus analysis of chronic postsurgical pain uncovers epigenetic mediators of genetic risk. Epigenomics. 2021;13(8):613-630. doi:10.2217/epi-2020-0424

24. Batoz H, Semjen F, Bordes-Demolis M, Benard A, Nouette-Gaulain $\mathrm{K}$. Chronic postsurgical pain in children: prevalence and risk factors. A prospective observational study. $B r \quad J$ Anaesth. 2016;117 (4):489-496. doi:10.1093/bja/aew260

25. Buvanendran A, Della Valle CJ, Kroin JS, et al. Acute postoperative pain is an independent predictor of chronic postsurgical pain following total knee arthroplasty at 6 months: a prospective cohort study. Reg Anesth Pain Med. 2019;44(3):e100036. doi:10.1136/rapm-2018-100036

26. Chow CHT, Schmidt LA, Buckley DN. The role of anxiety and related states in pediatric postsurgical pain. Can J Pain. 2020;4 (4):26-36. doi:10.1080/24740527.2020.1847600

27. Horn A, Kaneshiro K, Tsui BCH. Preemptive and preventive pain psychoeducation and its potential application as a multimodal perioperative pain control option: a systematic review. Anesth Analg. 2020;130(3):559-573. doi:10.1213/ANE.0000000000004319 
28. Rockett M, Creanor S, Squire R, et al. The impact of emergency department patient-controlled analgesia (PCA) on the incidence of chronic pain following trauma and non-traumatic abdominal pain. Anaesthesia. 2019;74(1):69-73. doi:10.1111/anae.14476

29. Capdevila X, Moulard S, Plasse C, et al. Effectiveness of epidural analgesia, continuous surgical site analgesia, and patient-controlled analgesic morphine for postoperative pain management and hyperalgesia, rehabilitation, and health-related quality of life after open nephrectomy: a prospective, randomized, controlled study. Anesth Analg. 2017;124(1):336-345.

30. Pan ZY, Hu ZH, Zhang F, Xie WX, Tang YZ, Liao Q. The effect of transversus abdominis plane block on the chronic pain after colorectal surgery: a retrospective cohort study. BMC Anesthesiol. 2020;20 (1):116. doi:10.1186/s12871-020-01032-8

31. Blichfeldt-Eckhardt MR. From acute to chronic postsurgical pain: the significance of the acute pain response. Dan Med J. 2018;65:3.

32. Pogatzki-Zahn E, Segelcke D, Zahn P. Mechanisms of acute and chronic pain after surgery: update from findings in experimental animal models. Curr Opin Anaesthesiol. 2018;31(5):575-585. doi:10.1097/ACO.0000000000000646

33. Gulur P, Nelli A. Persistent postoperative pain: mechanisms and modulators. Curr Opin Anaesthesiol. 2019;32(5):668-673. doi:10.1097/ACO.0000000000000770
34. Arboleda MF, Giron-Arango L, Peng PWH. Can recent chronic pain techniques help with acute perioperative pain? Curr Opin Anaesthesiol. 2019;32(5):661-667. doi:10.1097/ ACO.0000000000000772

35. Chapman CR, Vierck CJ. The transition of acute postoperative pain to chronic pain: an integrative overview of research on mechanisms. J Pain. 2017;18(4):359 e351-359 e338.

36. Theunissen M, Peters ML, Bruce J, Gramke HF, Marcus MA. Preoperative anxiety and catastrophizing: a systematic review and meta-analysis of the association with chronic postsurgical pain. Clin J Pain. 2012;28(9):819-841. doi:10.1097/AJP.0b013e31824549d6

37. Yang Y, Song Y, Zhang X, et al. Ketamine relieves depression-like behaviors induced by chronic postsurgical pain in rats through anti-inflammatory, anti-oxidant effects and regulating BDNF expression. Psychopharmacology (Berl). 2020;237(6):1657-1669. doi:10.1007/s00213-020-05490-3

38. Tinnirello A, Mazzoleni S, Santi C. Chronic pain in the elderly: mechanisms and distinctive features. Biomolecules. 2021;11 (8):1256. doi:10.3390/biom 11081256

39. Riley JL 3rd, Cruz-Almeida Y, Glover TL, et al. Age and race effects on pain sensitivity and modulation among middle-aged and older adults. J Pain. 2014;15(3):272-282. doi:10.1016/j.jpain.2013.10.015

\section{Video abstract}

\section{口的回

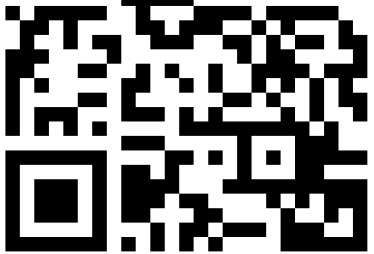

Point your SmartPhone at the code above. If you have a $Q R$ code reader the video abstract will appear. Or use: https://youtu.be//m3b]/s $Y$ wM4
International Journal of General Medicine

\section{Publish your work in this journal}

The International Journal of General Medicine is an international, peer-reviewed open-access journal that focuses on general and internal medicine, pathogenesis, epidemiology, diagnosis, monitoring and treatment protocols. The journal is characterized by the rapid reporting of reviews, original research and clinical studies

\section{Dovepress}

across all disease areas. The manuscript management system is completely online and includes a very quick and fair peer-review system, which is all easy to use. Visit http://www.dovepress.com/ testimonials.php to read real quotes from published authors. 